\title{
GEOMETRIC IDENTITIES IN STEREOLOGICAL PARTICLE ANALYSIS
}

\author{
STEPHAN KÖTZER \\ Department of Mathematical Statistics and Actuarial Science, University of Bern, Switzerland \\ e-mail: stephan.koetzer@stat.unibe.ch \\ (Accepted May 10, 2006)
}

\begin{abstract}
The paper reviews recent findings about geometric identities in integral geometry and geometric tomography, and their statistical application to stereological particle analysis. Open questions are discussed. This survey can also serve as an introduction to modern stereological particle analysis for readers who are interested in the mathematical background of the new methods.
\end{abstract}

Keywords: extremes, geometric identities, particle problem, shape modelling, stereology, Wicksell's corpuscle problem.

\section{INTRODUCTION}

Many identities in integral geometry and geometric tomography (Gardner, 1995) take the form

$$
\alpha(X)=\int \beta(X \cap T) d T
$$

where $X$ is a subset of some Euclidean space, $\alpha$ and $\beta$ are real valued functionals, $T$ ranges over a class of sets such as the $k$-dimensional planes, and typically $\mathrm{d} T$ represents integration with respect to an appropriate 'uniform' measure. The simplest example is the representation of the volume $\alpha(X)=V(X)$ of a three-dimensional solid $X$ as the integral of the areas $\beta(X \cap T)=A(X \cap T)$ of its horizontal plane sections $X \cap T$.

Such identities may be given a stochastic or statistical interpretation, which is the basis of the science of stereology (Baddeley and Jensen, 2005; Weibel, 1979; 1980). For example, the representation of volume in terms of the areas of plane sections was used by 19th century geologist A.E. Delesse to obtain a practical method for determining the composition of rocks. Namely, the volume percentage (fraction of volume of rock) occupied by a particular mineral of interest can be estimated from the fraction of area occupied by the same mineral in a single plane section of the rock. Note that this is different from applications to computed tomography, which require information from all plane sections of the object.

Until the 1980's, stereological techniques were based mainly on the classical section formulae of integral geometry, taking the form (Eq. 1), where $\alpha$ and $\beta$ are the intrinsic volumes or quermassintegrals in $\mathbb{R}^{d}$ for $d=1,2,3$, and $T$ is a $k$-dimensional plane, $k<d$. These formulae, and the stochastic interpretations that were placed on them, make it possible to statistically estimate volumes, areas and lengths of three-dimensional structures using information obtained from random plane sections of the structure (Weibel, 1979; 1980).

Stereologists use the generic term 'particles' to describe solid objects that can be scientifically interpreted as discrete individuals (such as biological cells, mineral grains, or enclosed holes). A 'particle population' is a finite or countable collection of disjoint particles.

The 'particle problem' in stereology is the problem of inferring the number, average size, and distribution of sizes, of a population of particles, from a plane section of the population. For example, given a microscope image of a plane section of brain tissue, we may wish to estimate the number of neurons in the brain, their average size, and the distribution of their sizes.

A general solution to the particle problem was not found in classical stereology. The classical identities of integral geometry (and their classical stochastic interpretation) only allow us to estimate 'aggregate' quantities $\alpha(X)$ where $X=\bigcup_{i} Y_{i}$ is the union of all the particles $Y_{i}$ in the population, and not 'individual' particle properties such as the distribution of particle sizes $\alpha\left(Y_{i}\right)$.

Until the 1980's, progress on the particle problem was achieved mainly by assuming that the particles have a common, simple, known shape such as spheres. Swedish mathematical statistician S.D. Wicksell first formulated the particle problem, and treated the case of estimating the size distribution of spheres in three dimensions from two-dimensional plane sections (Wicksell, 1925). In $\mathbb{R}^{n}$, the relation between the distribution function $F$ of the radii of the spheres 
and the distribution function $F^{(n, k)}$ of the radii of the spheres sectioned with a $k$-dimensional plane is given by, $c f$. Schneider and Weil (2000),

$$
\begin{aligned}
& F^{(n, k)}(x) \\
& =1-\frac{(n-k)}{M_{n-k}} \int_{x}^{\infty} u\left(u^{2}-x^{2}\right)^{\frac{n-k-2}{2}}(1-F(u)) d u,
\end{aligned}
$$

where $M_{n-k}$ is the $(n-k)$ th moment of sphere radii assumed positive and finite. Wicksell's problem has attracted considerable attention among mathematical statisticians, one reason being that it is an ill-posed problem implying that a small perturbation of the data in the section may lead to a large change in the estimated sphere size distribution.

Geometrical assumptions about particles - for example that the particles are all spheres - are overly simplistic for most applications. It is therefore a major advance that with the introduction of new sampling and measurement techniques such fragile geometrical assumptions can be relaxed. An example of these new techniques is 'local' sectioning of each particle through a reference point in the particle. Stereological methods based on such local sections constitute the new field of local stereology which is closely related to geometric tomography, $c f$. Gardner et al. (2003). Moments of particle size (including mean and variance) can be estimated stereologically without specific shape assumptions, using information on such local sections. (As a side remark, Wicksell's problem becomes trivial with such information at hand since the radius of a sphere can be observed directly in a section through the centre of the sphere.)

Prompted by the need to utilize the new type of data, new geometric identities have been discovered or rediscovered during the last couple of decades and have been used to renew stereological particle analysis. In the present paper we review these geometric identities in integral geometry and geometric tomography (Gardner, 1995) and point to some missing ones.

The first section describes the key geometrical identities. The second section discusses how the geometric identities can be used in the stereological analysis of particle populations. In section three we discuss inference about the particle size distribution. The fourth section describes approaches to modelling shapes. Finally in the last section we discuss some open problems.

An earlier review of stereology for geometers can be found in Weil (1983). Subsequently the development of three-dimensional microscopy, confocal microscopy and other imaging modes has led to a revolution in stereological sampling and measuring techniques. This involves both the development of new geometric identities, and alternative interpretations of existing geometric identities. A comprehensive treatment of stereology for statisticians has recently appeared in Baddeley and Jensen (2005), but for readers interested in the geometric background there seems to be no up-to-date review.

\section{GEOMETRIC IDENTITIES}

\section{THE BLASCHKE-PETKANTSCHIN FORMULAE}

The Blaschke-Petkantschin formulae play a fundamental role in modern stereological particle analysis. They provide the mathematical tool for estimating moments of particle volume from information on local $p$-dimensional sections through the particles.

The Blaschke-Petkantschin formulae are geometric measure decompositions of the $q$-fold product of Hausdorff measure in $\mathbb{R}^{n}$. In the special case of decomposition of one copy of $n$-dimensional Lebesgue measure $V_{n}$, the Blaschke-Petkantschin formula takes the following form for $X \subseteq \mathbb{R}^{n}$ compact and $p>r$ :

$$
\begin{aligned}
c(n-1- & r, p-1-r) V_{n}(X) \\
& =\int_{\mathscr{L}_{p(r)}^{n}} \int_{X \cap L_{p}}\left\|\pi_{L_{r}^{\perp}} x\right\|^{n-p} d x^{p} d L_{p(r)}^{n} .
\end{aligned}
$$

The normalizing constant is given by $c(n, p)=$ $\left(\sigma_{n} \sigma_{n-1} \cdots \sigma_{n-p+1}\right) /\left(\sigma_{p} \sigma_{p-1} \cdots \sigma_{1}\right)$, where $\sigma_{n}=$ $2(\pi)^{\frac{n}{2}} \Gamma\left(\frac{n}{2}\right)^{-1}$ is the surface area of the unit ball in $\mathbb{R}^{n}$. Note that by conventioyn $c(n, 0) \equiv 1$. The outer integration in (Eq. 3) is over the set $\mathscr{L}_{p(r)}^{n}$ of all $p$-dimensional linear subspaces $L_{p}$ (called for short $p$-subspaces in the following) of $\mathbb{R}^{n}$, containing a fixed $r$-subspace $L_{r}$, say. Note that $\mathscr{L}_{p(0)}^{n}=\mathscr{L}_{p}^{n}$, the set of $p$-subspaces in $\mathbb{R}^{n}$. The differentials $d x^{p}$ and $d L_{p(r)}^{n}$ are defined as $d x^{p}=\mathscr{H}_{p}^{n}(d x)$ and $d L_{p(r)}^{n}=$ $\mu_{p(r)}^{n}\left(d L_{p}\right)$, where $\mathscr{H}_{p}^{n}$ is $p$-dimensional Hausdorff measure in $\mathbb{R}^{n}$ and $\mu_{p(r)}^{n}$ is the unique measure on $\mathscr{L}_{p(r)}^{n}$ satisfying $\mu_{p(r)}^{n}\left(\mathscr{L}_{p(r)}^{n}\right)=c(n-r, p-r)$ such that $\mu_{p(r)}^{n}$ is invariant under rotations in $S O\left(n, L_{r}\right)=$ $\left\{B \in S O(n): B L_{r}=L_{r}\right\}$, the subgroup of rotations in $\mathbb{R}^{n}$, keeping the $r$-subspace $L_{r}$ fixed. Furthermore, the symbol $\|\cdot\|$ in (Eq. 3) denotes the Euclidean norm in $\mathbb{R}^{n}$ and $\pi_{L_{r}^{\perp}}$ is the orthogonal projection onto $L_{r}^{\perp}$, the orthogonal complement of $L_{r}$. 
More generally, suppose that $X_{1}, X_{2}, \ldots, X_{q}$ are compact subsets of $\mathbb{R}^{n}$. For $p \geq q+r$, we have

$$
\begin{array}{r}
c(n-q-r, p-q-r) V_{n}\left(X_{1}\right) \cdots V_{n}\left(X_{q}\right) \\
=\int_{\mathscr{L}_{p(r)}^{n}} \int_{X_{1} \cap L_{p}} \cdots \int_{X_{q} \cap L_{p}} \nabla_{q}\left(\pi_{L_{r}^{\perp}} x_{1}, \ldots, \pi_{L_{r}^{\perp}} x_{q}\right)^{n-p} \times \\
\times \prod_{i=1}^{q} d x_{i}^{p} d L_{p(r)}^{n},
\end{array}
$$

where $\nabla_{q}\left(x_{1}, \ldots, x_{q}\right)=q ! \mathscr{H}_{q}^{n}\left(\operatorname{conv}\left\{O, x_{1}, \ldots, x_{q}\right\}\right)$ and conv $\left\{O, x_{1}, \ldots, x_{q}\right\}$ denotes the convex hull of the set $\left\{O, x_{1}, \ldots, x_{q}\right\}$. Here, the point $O \in \mathbb{R}^{n}$ is the origin of $\mathbb{R}^{n}$.

Early versions of the Blaschke-Petkantschin formulae for decomposition of Lebesgue measures are due to Blaschke (1935a;b) and Petkantschin (1936). The one presented above has been derived in Miles (1979). The general decomposition of the $q$-fold product of $d$-dimensional Hausdorff measure in $\mathbb{R}^{n}$ has been established in Zähle (1990) and Jensen and Kiêu (1992). For $d=n$ the decomposition reduces to the one presented above while for $d<n$ it involves the so-called $G$-factors which contain information about the angle between the boundary of the sets $X_{i}$ and the intersecting $p$-subspace.

The formula for decomposition of Lebesgue measures has been proved by invariant measure theory (Møller, 1987) while the general formula for $d$ dimensional Hausdorff measures has been established using geometric measure theory, see Zähle (1990) and Jensen and Kiêu (1992). A simplified proof, also utilizing induction in the dimension of the spaces involved, can be found in Jensen (1998, Ch. 5.4).

\section{A LOCAL SLICE FORMULA}

For $d<n$, the Blaschke-Petkantschin formulae depend on angles defined in $\mathbb{R}^{n}$ which cannot be determined only from information on lowerdimensional sections $X \cap L_{p}$. Also in the case $d=0$ the decomposition is trivial so it is not possible to get information about number using the BlaschkePetkantschin formulae. This has been the motivation for deriving geometric identities for $p$-dimensional slices centred at a reference point. Such slices are $p$ subspaces with some thickness.

The local slice formula gives a geometric decomposition of $d$-dimensional Hausdorff measure in $\mathbb{R}^{n}$ for $d=0,1, \ldots, n$. A $p$-slice $T_{p}$ of thickness $2 t$ is a set of the form $L_{p}+B^{n}(O, t)$, where $B^{n}(O, t)=\{x \in$ $\left.\mathbb{R}^{n}:|| x \|<t\right\}$ is the open ball in $\mathbb{R}^{n}$ with centre $O \in \mathbb{R}^{n}$ and radius $t>0$. With $\mathscr{T}_{p(r)}^{n}$ we denote the set of $p$ slices $L_{p}+B^{n}(O, t)$ for which $L_{p} \supseteq L_{r}$, where $L_{r}$ is a fixed $r$-subspace. Similar as in the subsection before the differential $d T_{p(r)}^{n}$ is defined as $d T_{p(r)}^{n}=v_{p(r)}^{n}\left(d T_{p}\right)$, where $v_{p(r)}^{n}$ is the unique measure on $\mathscr{T}_{p(r)}^{n}$ satisfying $v_{p(r)}^{n}\left(\mathscr{T}_{p(r)}^{n}\right)=c(n-r, p-r)$ such that $v_{p(r)}^{n}$ is invariant under rotations in $S O\left(n, L_{r}\right)$. Let $X$ be a $d$-dimensional differentiable and bounded manifold in $\mathbb{R}^{n}$. Then, for arbitrary $p, r$ satisfying $0 \leq r<p<n$, the local slice formula takes the form

$$
\begin{aligned}
& c(n-r, p-r) V_{n}^{d}(X) \\
& =\int_{\mathscr{T}_{p(r)}^{n}} \int_{X \cap T_{p}} h_{p(r)}^{(n)}\left(x, L_{r}\right)^{-1} d x^{d} d T_{p(r)}^{n},
\end{aligned}
$$

where

$$
h_{p(r)}^{(n)}\left(x, L_{r}\right)=F_{n-p, p-r}\left(t^{2} /\left\|\pi_{L_{r}^{\perp}} x\right\|^{2}\right)
$$

and $F_{a, b}$ is the distribution function of the Beta distribution with parameters $(a / 2, b / 2)$.

An early version of this geometric identity can be found in Jensen and Kiêu (1994). The proof of the slice identity uses the following reasoning that holds for any $x \in \mathbb{R}^{n}$.

$$
\begin{aligned}
\int_{\mathscr{T}_{p(r)}^{n}} \mathbf{1}\left\{x \in T_{p}\right\} d T_{p(r)}^{n} \\
=\int_{\mathscr{L}_{p(r)}^{n}} \mathbf{1}\left\{\left\|\pi_{L_{p}^{\perp}} x\right\|<t\right\} d L_{p(r)}^{n} \\
=c(n-r, p-r) F_{n-p, p-r}\left(t^{2} /\left\|\pi_{L_{r}^{\perp}} x\right\|^{2}\right) .
\end{aligned}
$$

At the last equality sign it is used that the length of the isotropic projection of a line segment is Beta distributed. The more general issue of isotropic projections of simplices has been studied in Nielsen (1999). Here the distribution becomes that of a product of independent Beta-distributed random variables.

\section{A GEOMETRIC IDENTITY INVOLVING VERTICAL SECTIONS}

Vertical sections are hyperplanes parallel to a fixed axis. Such sections are often used in microscopy to reveal structural information or for practical convenience. In the 1980s, Baddeley (1984; 1985; 1987) and collaborators (Baddeley et al., 1986) showed how a rotation invariant line in $\mathbb{R}^{n}$ can be generated via a vertical section. The important consequence is that results based on rotation invariant lines can be transformed into results for lines generated on vertical sections. We will in this subsection present this key result and show how it can be used to derive an alternative local geometric identity for volume. The main application of Baddeley's result has, however, 
been in the development of a stereological estimator for surface area. This estimator has also relevance for stereological particle analysis, as shown in section two. Another important issue are vertical probes, which have been developed to vertical projections by Gokhale (1990). We do not consider vertical probes in this paper but for the interested reader we refer to a survey in Beneš and Rataj (2004).

Let $\mathscr{L}_{n-1(1)}^{n}$ be the set of hyperplanes through $O$ containing $v \in S^{n-1}$, where $S^{n-1}=\left\{x \in \mathbb{R}^{n}:\|x\|=1\right\}$. Then, the key result proved by Baddeley can be stated as follows:

$$
\begin{aligned}
& \int_{\mathscr{L}_{1}^{n}} h\left(L_{1}\right) d L_{1}^{n} \\
& \quad=\int_{\mathscr{L}_{n-1(1)}^{n}} \int_{L_{1} \subseteq L_{n-1}} h\left(L_{1}\right) g_{1}^{n-2}\left(L_{1}, v\right) d L_{1}^{n-1} d L_{n-1(1)}^{n},
\end{aligned}
$$

where $g_{1}(L, v)=|\sin \angle(L, v)|$. In Beneš and Rataj (2004, Ch. 4.1.3), (Eq. 4) has been proved by first establishing the following identity, using the coarea formula and spherical coordinates,

$$
\begin{aligned}
& \int_{S^{n-1}} f(u) d u^{n-1} \\
& \quad=\int_{S^{n-2}\left(v^{\perp}\right)} \int_{S_{+}^{1}(\operatorname{span}\{l, v\})} f(u) g_{2}^{n-2}(u, v) d u^{1} d l^{n-2},
\end{aligned}
$$

where $S^{n-2}\left(v^{\perp}\right)$ is the unit ball in the hyperplane $v^{\perp}$ through the origin, perpendicular to $v, \operatorname{span}\{l, v\}$ is the linear subspace of $\mathbb{R}^{n}$, spanned by $l$ and $v, S_{+}^{1}=\{\omega=$ $\left.\left(\omega_{1}, \omega_{2}\right) \in S^{1}: \omega_{2}>0\right\}$, and $g_{2}(u, v)=|\sin \angle(u, v)|$. See Fig. 1 for an illustration in 3D. Note that (Eq. 4) is indeed implied by (Eq. 5) since

$$
\begin{array}{rl}
\int_{\mathscr{L}_{1}^{n}} & h\left(L_{1}\right) d L_{1}^{n} \\
& =\frac{1}{2} \int_{S^{n-1}} h(\operatorname{span}\{u\}) d u^{n-1} \\
& =\frac{1}{2} \int_{S^{n-2}\left(v^{\perp}\right)} \int_{S_{+}^{1}(\operatorname{span}\{l, v\})} h(\operatorname{span}\{u\}) g_{2}^{n-2}(u, v) d u^{1} d l^{n-2} \\
& =\int_{S_{+}^{n-2}\left(v^{\perp}\right)} \int_{S_{+}^{1}(\operatorname{span}\{l, v\})} h(\operatorname{span}\{u\}) g_{2}^{n-2}(u, v) d u^{1} d l^{n-2} \\
& =\int_{\mathscr{L}_{n-1(1)}^{n}} \int_{L_{1} \subseteq L_{n-1}} h\left(L_{1}\right) g_{2}^{n-2}(u, v) d L_{1}^{n-1} d L_{n-1(1)}^{n} .
\end{array}
$$

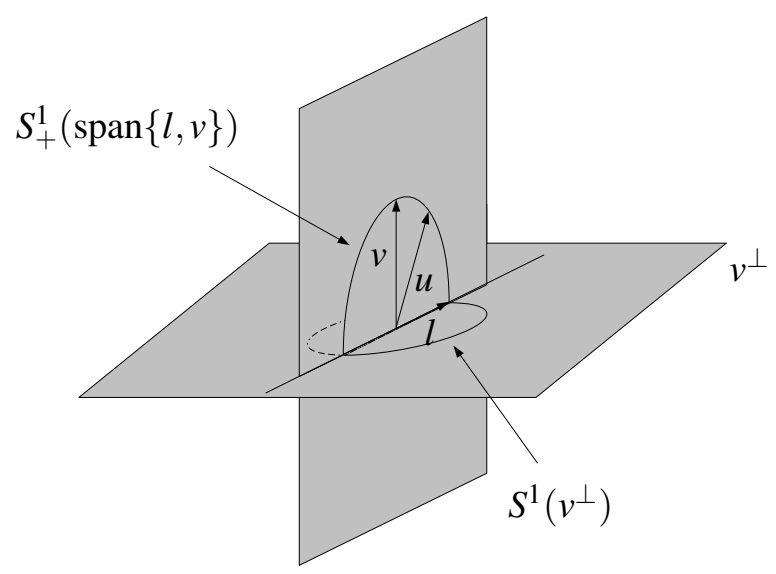

Fig. 1. Illustration relevant for the identity (Eq. 5), relating to vertical sectioning.

As announced in the beginning of this subsection, Eq. 4 has been used to derive a local identity for volume. Combining with the Blaschke-Petkantschin formula (Eq. 3) with $p=1$ and $r=0$, we get

$$
\begin{aligned}
V_{n}(X) & =\int_{\mathscr{L}_{1}} \int_{X \cap L_{1}}\|x\|^{n-1} d x^{1} d L_{1}^{n} \\
& =\int_{\mathscr{L}_{n-1(1)}^{n}} \beta\left(X \cap L_{n-1}\right) d L_{n-1(1)}^{n},
\end{aligned}
$$

where

$$
\begin{aligned}
\beta(X & \left.\cap L_{n-1}\right) \\
= & \int_{L_{1} \subseteq L_{n-1}} \int_{X \cap L_{1}}\|x\|^{n-1} g_{1}^{n-2}\left(L_{1}, v\right) d x^{1} d L_{1}^{n-1} .
\end{aligned}
$$

\section{A GEOMETRIC IDENTITY FOR SURFACE AREA}

The Blaschke-Petkantschin formulae depend for $d<n$ on angles in $\mathbb{R}^{n}$ which cannot be determined on a lower dimensional section. As an example, for an $(n-1)$-dimensional manifold $X$ we have

$$
\begin{aligned}
& c(n-1, p-1) S_{n}(X) \\
& \quad=\int_{\mathscr{L}_{p}^{n}} \int_{X \cap L_{p}}\|x\|^{n-p} / G\left(\operatorname{Tan}[X, x], L_{p}\right) d L_{p}^{n},
\end{aligned}
$$

where $S_{n}=\mathscr{H}_{n-1}^{n}$ denotes surface area in $\mathbb{R}^{n}$, $G\left(L_{d}, L_{p}\right)=\left|\sin \alpha_{d, p}\right|$ and $\alpha_{d, p}$ is a generalized angle between the subspaces $L_{d}$ and $L_{p}$. Furthermore, $\operatorname{Tan}[X, x]$ is the tangent space of $X$ at $x \in X$. In Jensen (1998, Ch. 5.6), it is shown that the geometric identity can be modified such that only lower-dimensional 
angular information is used. The modified geometric identity is of the following form:

$$
c(n-1, p-1) S_{n}(X)=\int_{\mathscr{L}_{p}^{n}} \beta\left(X \cap L_{p}\right) d L_{p}^{n},
$$

where

$$
\beta\left(X \cap L_{p}\right)=\int_{\mathscr{L}_{1}^{p}} \sum_{x \in X \cap L_{1}}\|x\|^{n-1} h\left(\tan \beta_{L_{1}}(x)\right) d L_{1}^{p},
$$

$\beta_{L_{1}}(x)$ is the angle between $L_{1}$ and the unit normal vector of the tangent space of $X \cap L_{p}$ at $x$, and $h$ can be expressed as a hypergeometric function

$$
h(u)=F\left(-\frac{1}{2}, \frac{1}{2}(n-1) ; \frac{1}{2}(p-1) ;-u^{2}\right) .
$$

Very recently, an alternative approach to estimate surface area has been suggested by Cruz-Orive (2005). The method is based on a new principle to generate isotropic uniform random test lines hitting a particle.

\section{STEREOLOGICAL PARTICLE ANALYSIS}

We will now discuss how the geometric identities can be used in the stereological analysis of particle populations.

\section{STOCHASTIC MODEL}

A 'particle' is a compact, nonempty subset of $\mathbb{R}^{n}$. For instance, the cells in a biological tissue or the inclusions in steel may be modelled as a collection of particles in $\mathbb{R}^{3}$. To study the particle problem we must consider the intersection between a collection of particles and a 'probe' $T$ such as a plane or a line. Either $T$ is randomly positioned (a "design-based" approach) or the collection of particles is random (a "model-based" approach). In this review we focus on the model-based approach.

D. Stoyan, J. Mecke and collaborators (Hanisch and Stoyan, 1981; Mecke and Stoyan, 1980; Stoyan, 1979; 1982; 1990; 1996; Stoyan and Mecke, 1983) formulated the particle problem using the theory of point processes (Daley and Vere-Jones, 1988). First consider the very simple case of a collection of spheres $B\left(x_{i}, r_{i}\right)$ in $\mathbb{R}^{3}$ with random centres $x_{i} \in \mathbb{R}^{3}$ and random radii $r_{i}>0$. The collection of pairs $\left(x_{i}, r_{i}\right)$ can be treated as a point process in $\mathbb{R}^{3} \times \mathbb{R}_{+}$. Equivalently this is a 'marked point process' in $\mathbb{R}^{3}$ with marks in $\mathbb{R}_{+}$.

A marked point process in $\mathbb{R}^{n}$ with marks in some space $\mathscr{M}$ can be defined formally as a point process in $\mathbb{R}^{n} \times \mathscr{M}$ satisfying certain conditions. It is interpreted as a point process in $\mathbb{R}^{n}$ where each point $x_{i} \in \mathbb{R}^{n}$ of the process carries additional information in the form of a mark $m_{i} \in \mathscr{M}$. For details see e.g., Stoyan et al. (1987).

The class $\mathscr{K}$ of all particles is a complete separable metric space, so a random particle (a random element of $\mathscr{K}$ ) can be defined in a straightforward manner (Matheron, 1975). A random collection of particles is most conveniently modelled as a marked point process in $\mathbb{R}^{n}$ with marks in $\mathscr{K}$.

The particles are regarded as a realization of a marked point process $\Psi=\left\{\left[x_{i} ; \Xi_{i}\right]\right\}$ in $\mathbb{R}^{n}$, where the $x_{i}$ 's are points in $\mathbb{R}^{n}$ and the marks $\Xi_{i}$ are compact subsets of $\mathbb{R}^{n}$. The $i$ th particle of the process is represented by the set $X_{i}=x_{i}+\Xi_{i}$. In this framework, $x_{i}$ is called the nucleus of the $i$ th particle and $\Xi_{i}$ the 'primary' or 'centred' particle. The corresponding process of nuclei is denoted by $\Psi_{n}=\left\{x_{i}\right\}$.

The marked point process $\Psi$ is assumed to be stationary, i.e., for all $x \in \mathbb{R}^{n}$ we have $\Psi_{x} \sim \Psi$, where $\Psi_{x}=\left\{\left[x_{i}+x ; \Xi_{i}\right]\right\}$. Furthermore, in some cases we assume that $\Psi$ is isotropic, i.e., the distribution of $\Psi$ is invariant under rotations $A$, meaning that $A \Psi=$ $\left\{\left[A x_{i} ; A \Xi_{i}\right]\right\}$ coincides in distribution with $\Psi$.

Since $\Psi$ is stationary, the intensity measure of $\Psi$ can be written as

$$
\begin{aligned}
& \Lambda(B \times K) \\
& \quad=\mathbf{E} \sum_{i} \mathbf{1}\left\{x_{i} \in B, \Xi_{i} \in K\right\}=V_{n}(B) N_{V}(K),
\end{aligned}
$$

for $B \in \mathscr{B}\left(\mathbb{R}^{n}\right)$ and $K \in \mathscr{K}$. Here $\mathscr{B}\left(\mathbb{R}^{n}\right)$ is the Borel $\sigma$-algebra in $\mathbb{R}^{n}$ and $N_{V}(K)$ is the mean number of particles per unit volume with marks in $K$. The mark distribution is defined as

$$
\mathbf{P}_{m}(K)=\frac{N_{V}(K)}{N_{V}}, \quad K \in \mathscr{K}
$$

where $N_{V} \in(0, \infty)$ is the intensity of $\Psi_{n}$. Using Eq. 6 and Eq. 7 it can be shown for any measurable nonnegative function $h$ defined on $\mathbb{R}^{n} \times \mathscr{K}$,

$$
\mathbf{E} \sum_{i} h\left(x_{i}, \Xi_{i}\right)=N_{V} \int_{\mathbb{R}^{n}} \int_{\mathscr{K}} h(x, K) \mathbf{P}_{m}(d K) d x .
$$

In what follows, we let $\Xi_{0}$ be a random compact set with distribution $\mathbf{P}_{m}$. If $\Psi$ is isotropic, then $A \Xi_{0} \sim \Xi_{0}$ for all rotations $A$. 


\section{GEOMETRIC SAMPLING EFFECTS}

When a population of particles is sectioned by a plane, two 'sampling effects' occur. First there is sizedependent sampling bias: the probability that a given particle is hit by the section plane depends on the size of the particle. Second, there is random reduction in size: a plane section of a particle is smaller than the particle itself.

These effects were first explained by Wicksell (1925) in the case of spherical particles. Consider a stationary process of spheres in $\mathbb{R}^{3}$, intersected by a fixed two-dimensional plane. The plane section is a stationary process of circles in $\mathbb{R}^{2}$. Size-dependent sampling bias occurs because, roughly speaking, the probability that a given sphere is intersected by the section plane is proportional to the sphere's radius. Let $N_{V}$ denote the expected number of sphere centres per unit volume in the population, and $N_{A}$ the expected number of circle centres per unit area in the plane section. Then $N_{V}$ and $N_{A}$ are not equal, but instead are related by

$$
N_{A}=N_{V} \mathbf{E} D
$$

where $\mathbf{E} D$ is the mean sphere diameter in the original particle process. If the sphere radii have cumulative distribution function $F$, then the spheres selected by a random section plane have radii with distribution function

$$
F_{1}(r)=\frac{1}{\mathbf{E} R} \int_{0}^{r} s d s
$$

the size-biased counterpart of $F$. Here, $\mathbf{E} R$ denotes the mean sphere radius in the original particle process.

For particles of more general shape, it was established by DeHoff and Rhines (1961) and others, then in complete generality by Stoyan (1979), that

$$
N_{V}=N_{A} \mathbf{E} H
$$
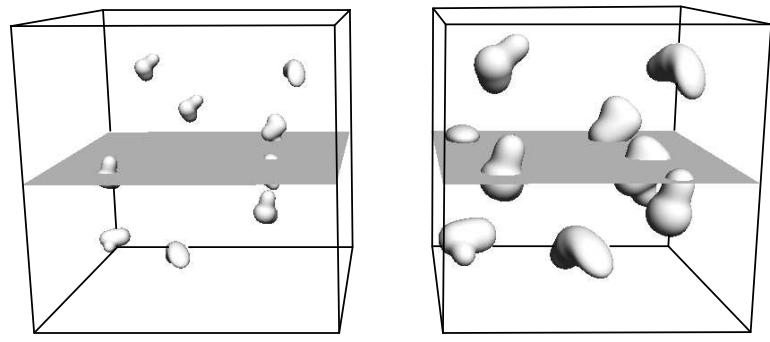

Fig. 2. An explanation that $Q$ must depend on the mean particle height $\mathbf{E} H$. The two boxes contain equal numbers of particles. The particles on the left are smaller than those on the right. A typical section plane cuts fewer particles in the left box than it does on the right. where $N_{V}$ is the intensity of the particle process (expected number of particle centres $x_{i}$ per unit volume), $N_{A}$ is the intensity of the process of section profiles (the expected number of particle profiles per unit area in the plane section) and $\mathbf{E} H$ is the mean particle height. The height $H$ of a particle is the length of its projection onto the subspace normal to the section plane. Relation (Eq. 11) is known as the 'Rhines-DeHoff equation'. See Fig. 2 for an illustration in $3 \mathrm{D}$.

\section{PARTICLE NUMBER}

An important task in stereology is the estimation of $N_{V}$, the mean number of particles per unit volume of the material of interest. Unfortunately, it is not possible to estimate $N_{V}$, in complete generality, from a plane section alone. This can be seen from the Rhines-DeHoff relation (Eq. 11). The population average height of the particles $\mathbf{E} H$ cannot generally be determined from a plane section. The problem can be circumvented only when we have extra information, for example when we have some knowledge about the particle shape, or when particle height measurements are available.

In order to construct an unbiased estimator for $N_{V}$ in a situation where the particles are of general shape, we need a different sampling scheme. Consider a sampling scheme with the property that for any fixed sampling window $W \in \mathscr{K}$ with positive volume and any particle $X \in \mathscr{K}$,

$$
V_{n}\left(\left\{u \in \mathbb{R}^{n}: X \text { sampled in } u+W\right\}\right)=V_{n}(W) .
$$

Let

$$
\mathscr{S}=\left\{i: X_{i} \text { sampled in } W\right\} .
$$

Then, using Eq. 8 and Eq. 12, we conclude that

$$
\begin{aligned}
\mathbf{E} N(\mathscr{S}) & =\mathbf{E} \sum_{i} \mathbf{1}\left\{X_{i} \text { sampled in } W\right\} \\
= & N_{V} \int_{\mathbb{R}^{n}} \int_{\mathscr{K}} \mathbf{1}\{x+K \text { sampled in } W\} \mathbf{P}_{m}(d K) d x \\
= & N_{V} \int_{\mathscr{K}} \int_{\mathbb{R}^{n}} \mathbf{1}\{K \text { sampled in } W-x\} d x \mathbf{P}_{m}(d K) \\
= & N_{V} V_{n}(W) .
\end{aligned}
$$

Therefore an unbiased estimator for $N_{V}$ is given by

$$
\widehat{N}_{V}=\frac{N(\mathscr{S})}{V_{n}(W)} .
$$

In practice Miles' associated point rule (Miles, 1974) and Gundersen's tiling rule (Gundersen, 1977; 1978) satisfy the basic requirement (Eq. 12). See Fig. 3 for an illustration in $2 \mathrm{D}$ of these two sampling rules. 

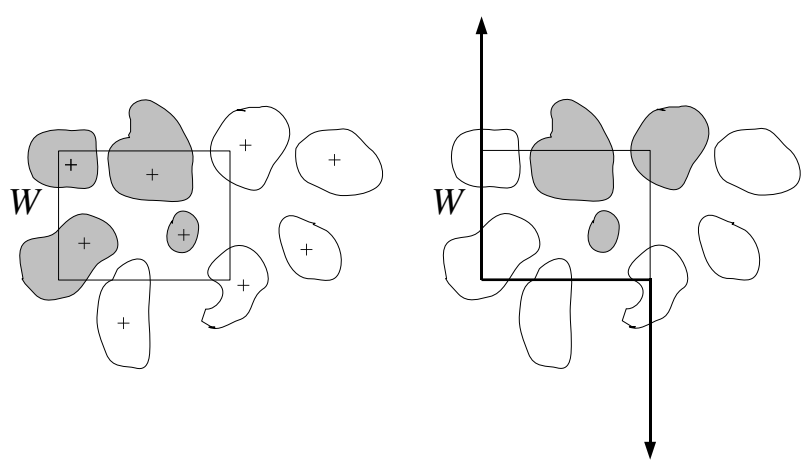

Fig. 3. Left: associated point rule, profiles with associated point in the window are sampled. Right: tiling rule, profiles intersecting $W$ but not the thick lines extending to infinity are sampled.

\section{MOMENTS OF PARTICLE SIZE}

The geometric identities presented in the first section can be used to derive estimators of moments of particle size $\mathbf{E} \varphi\left(\Xi_{0}\right)^{q}, q \geq 1$, where $\varphi$ is a selected size parameter. Below we give two examples of such estimators.

\section{Estimators based on the Blaschke- \\ Petkantschin formula}

Suppose that the distribution of $\Xi_{0}$ is invariant under the action of $S O\left(n, L_{r}\right)$. Using Eq. 3, we get

$$
\begin{aligned}
& c(n-1-r, p-1-r) \mathbf{E} V_{n}\left(\Xi_{0}\right) \\
& \quad=\int_{\mathscr{L}_{p(r)}^{n}}\left[\mathbf{E} \int_{\Xi_{0} \cap L_{p}}\left\|\pi_{L_{r}^{\perp}} x\right\|^{n-p} d x^{p}\right] d L_{p(r)}^{n} .
\end{aligned}
$$

Since the distribution of $\Xi_{0}$ is invariant under rotations around $L_{r}$, the inner integral of the right-hand side of (Eq. 13) does not depend on $L_{p}$ and we get for a particular $p$-subspace $L_{p 0} \in \mathscr{L}_{p(r)}^{n}$,

$$
\mathbf{E} V_{n}\left(\Xi_{0}\right)=\frac{\sigma_{n-r}}{\sigma_{p-r}} \mathbf{E} \int_{\Xi_{0} \cap L_{p 0}}\left\|\pi_{L_{r}^{\perp}} x\right\|^{n-p} d x^{p} .
$$

It follows that

$$
\frac{\sigma_{n-r}}{\sigma_{p-r}} \int_{\Xi_{0} \cap L_{p 0}}\left\|\pi_{L_{r}^{\perp}} x\right\|^{n-p} d x^{p}
$$

is an unbiased estimator of $\mathbf{E} V_{n}\left(\Xi_{0}\right)$.

In practice, a sample of particles $\left\{x_{i}+\Xi_{i}: x_{i} \in W\right\}$ is collected in a sampling window $W$ and a central section

$$
\left(x_{i}+\Xi_{i}\right) \cap\left(x_{i}+L_{p 0}\right)
$$

is determined through each particle. An illustration of this sampling scheme for $n=2$ and $p=1$ is shown in Fig. 4. For each sampled particle, we determine

$$
h\left(x_{i}, \Xi_{i}\right)=\frac{\sigma_{n-r}}{\sigma_{p-r}} \int_{\left(x_{i}+\Xi_{i}\right) \cap\left(x_{i}+L_{p 0}\right)}\left\|\pi_{L_{r}^{\perp}}\left(x-x_{i}\right)\right\|^{n-p} d x^{p} .
$$

Using Eq. 8, we find that

$$
\begin{aligned}
& \mathbf{E} \sum_{\left\{i: x_{i} \in W\right\}} h\left(x_{i}, \Xi_{i}\right) \\
& \quad=N_{V} V_{n}(W) \frac{\sigma_{n-r}}{\sigma_{p-r}} \mathbf{E} \int_{\Xi_{0} \cap L_{p 0}}\left\|\pi_{L_{r}^{\perp}} x\right\|^{n-p} d x^{p} \\
& \quad=N_{V} V_{n}(W) \mathbf{E} V_{n}\left(\Xi_{0}\right) .
\end{aligned}
$$

We can thereby construct an unbiased estimator of $\mathbf{E} V_{n}\left(\Xi_{0}\right)$ if an estimate of $N_{V}$ can be constructed.

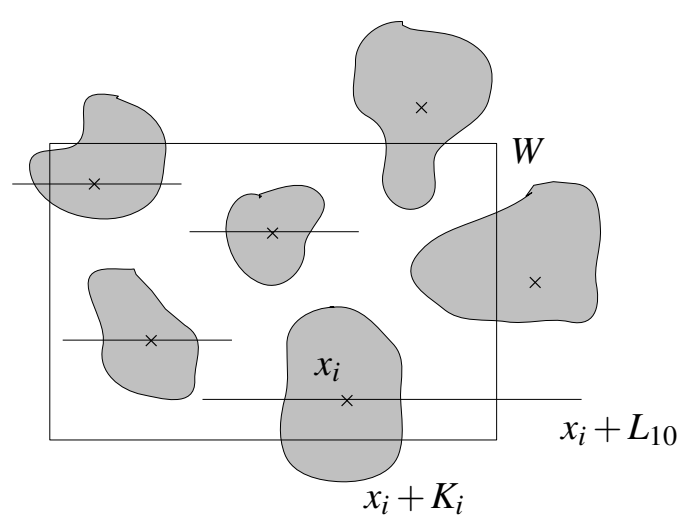

Fig. 4. Local sampling scheme. Particles $x_{i}+\Xi_{i}$ satisfying $x_{i} \in W$ are sampled using a fixed line $L_{10}$.

\section{Estimators based on vertical sections}

In the same way, other identities from the first section can be used to construct estimators of $\mathbf{E} V_{n}\left(\Xi_{0}\right)^{q}$ or $\mathbf{E} S_{n}\left(\Xi_{0}\right)$.

The identity (Eq. 4) involving vertical sections provides an alternative for estimating $\mathbf{E} S_{n}\left(\Xi_{0}\right)$ if the distribution of $\Xi_{0}$ is invariant under rotations around the vertical axis $v$. The identity is used in combination with the classical Crofton formula. Let us suppose that the particles are non-overlapping and let

$$
Z=\bigcup_{i}\left(x_{i}+\Xi_{i}\right) .
$$

Then, using (Eq. 8) we find that

$$
\mathbf{E} S_{n}(Z \cap W)=N_{V} V_{n}(W) \mathbf{E} S_{n}\left(\Xi_{0}\right) .
$$


We now use one of the Crofton formulae for Hausdorff rectifiable closed sets, stating that

$$
\begin{aligned}
& S_{n}(Z \cap W) \\
& =\frac{1}{\omega_{n-1}} \int_{\mathscr{L}_{1}^{n}} \int_{L_{1}^{\perp}} N\left(Z \cap W \cap\left(L_{1}+t\right)\right) d t^{n-1} d L_{1}^{n},
\end{aligned}
$$

where $\omega_{n-1}=\pi^{\frac{n-1}{2}} \Gamma\left(\frac{n-1}{2}+1\right)^{-1}$ is the Lebesgue measure of the unit ball in $\mathbb{R}^{n-1}$ and $N(\cdot)=\mathscr{H}_{0}^{n}(\cdot)$ denotes counting measure. The stationarity of $Z$ implies

$$
\mathbf{E} N\left(Z \cap W \cap\left(L_{1}+t\right)\right)=N_{L}\left(L_{1}\right) L_{n}\left(W \cap\left(L_{1}+t\right)\right),
$$

where $N_{L}\left(L_{1}\right)$ is the average number of intersection points per unit length of test lines in direction $L_{1}$ and $L_{n}(\cdot)=\mathscr{H}_{1}^{n}(\cdot)$ denotes length in $\mathbb{R}^{n}$. Hence, using Fubini's theorem and (Eq. 4), we conclude that

$$
\begin{aligned}
\mathbf{E} S_{n} & (Z \cap W) \\
= & \frac{1}{\omega_{n-1}} \int_{\mathscr{L}_{1}^{n}} N_{L}\left(L_{1}\right) \int_{L_{1}^{\perp}} L_{n}\left(W \cap\left(L_{1}+t\right)\right) d t^{n-1} d L_{1}^{n} \\
= & \frac{1}{\omega_{n-1}} V_{n}(W) \int_{\mathscr{L}_{1}^{n}} N_{L}\left(L_{1}\right) d L_{1}^{n} \\
= & \frac{1}{\omega_{n-1}} V_{n}(W) \int_{\mathscr{L}_{n-1(1)}^{n}(v)} \int_{L_{1} \subseteq L_{n-1}} N_{L}\left(L_{1}\right) \times \\
& \times g_{1}^{n-2}\left(L_{1}, v\right) d L_{1}^{n-1} d L_{n-1(1)}^{n} .
\end{aligned}
$$

Because of the rotational invariance of $Z$ around the vertical axis $v, N_{L}\left(L_{1}\right)$ only depends on $\angle\left(L_{1}, v\right)$, $N_{L}\left(L_{1}\right)=N_{L}\left(\angle\left(L_{1}, v\right)\right)$, say, and the inner integral of Eq. 15 does not depend on $L_{n-1(1)}^{n}$. Choosing a particular vertical plane $L_{n-1,0}$ we get, using Eq. 14,

$$
\begin{aligned}
& \mathbf{E} S_{n}\left(\Xi_{0}\right)=\mathbf{E} \frac{S_{n}(Z \cap W)}{N_{V} V_{n}(W)} \\
& =\frac{1}{N_{V}} \frac{\sigma_{n-1}}{\omega_{n-1}} \int_{L_{1} \subseteq L_{n-1,0}} N_{L}\left(\angle\left(L_{1}, v\right)\right) g_{1}^{n-2}\left(L_{1}, v\right) d L_{1}^{n-1} .
\end{aligned}
$$

After normalization, the integral can be interpreted as the mean number of intersection points per unit length of test line for a random line $L_{1}$ with a density with respect to $d L_{1}^{n-1}$ proportional to $\left|\sin \angle\left(L_{1}, v\right)\right|^{n-2}$.

\section{INFERENCE ON THE PARTICLE SIZE DISTRIBUTION}

At present, there exists no method of obtaining the entire particle size distribution from sections without assumptions about particle shape.
If the particles are spheres, Eq. 2 gives the relation between the distribution function $F$ of sphere radii and the distribution function $F^{(n, k)}$ of radii of sectioned spheres on a $k$-dimensional affine subspace. A proof of Eq. 2 can be found in Schneider and Weil (2000, p. 144-145) for a stationary marked point process of spheres, intersected by a fixed $k$-dimensional affine subspace. Note that the point process of spheres centres need not follow a Poisson process.

For particles of varying and general shape, $\mathbf{E} \varphi\left(\Xi_{0}\right)$ and $\mathbf{E} \varphi\left(\Xi_{0}\right)^{2}$ can be estimated from sections as explained in the second section for selected size parameters $\varphi$. The variance of $\varphi\left(\Xi_{0}\right)$ can therefore be estimated. If the variance in the distribution of estimated sizes is close to the variance in the distribution of true sizes, then the distribution of estimated sizes will be close to the true size distribution. The potential of this reasoning is investigated in Jensen and Pawlas (2005).

In recent years, there has been an increasing interest in estimating stereologically the tail of the particle size distribution. From a practical point of view, this is clearly of interest since the property of a material may be related to the extreme particle sizes rather than the mean particle size. For example, in the production of clean steels the occurrence of imperfections - so-called inclusions - cannot be avoided. Furthermore, the fatigue strength of a block of clean steel is largely dependent on the size of the largest inclusions it contains, so inference on extreme inclusion size forms an important part of quality control. The data is usually collected on planar slices which leads to an extreme value version of a stereological problem: how to predict extremal size from measurements on planar slices. In the following we give a brief introduction into this relatively new field of stereology of extremes; see also Beneš and Rataj (2004) for a more detailed survey.

Extreme value theory has been used to get information about the tail of the particle size distribution in the case of spherical particles, under the assumption that the radii of the spheres are i.i.d. and independent of the process of sphere centres. Let $\left\{Y_{i}, i \geq 1\right\}$ be the sequence of independent and identically distributed sphere radii with common distribution function $F$. Denote the sample maximum by

$$
Z_{n}=\max \left(Y_{1}, \ldots, Y_{n}\right), \quad n \geq 1 .
$$

If there are sequences of normalizing constants $a_{n}>0$ and $b_{n} \in \mathbb{R}$ such that the normalized maxima $\left(Z_{n}-\right.$ $\left.b_{n}\right) / a_{n}$ converge in distribution to a nondegenerate distribution function $M$, then $F$ lies in the domain of attraction of $M$. We then write $F \in \mathscr{D}(M)$. There are 
only three types of possible limiting distributions $M$ (de Haan, 1975; Galambos, 1987), namely

$$
M_{i, \alpha}(x)=\left\{\begin{array}{lll}
\exp \left(-x^{-\alpha}\right) & x \geq 0, \quad i=1, \\
\exp \left(-(-x)^{\alpha}\right) & x \leq 0, \quad i=2,
\end{array} \quad \alpha>0,\right.
$$

and

$$
M_{3}(x)=\exp \left(-e^{-x}\right), \quad x \in \mathbb{R} .
$$

$M_{1, \alpha}, M_{2, \alpha}$ and $M_{3}$ are called Fréchet, Weibull and Gumbel distributions respectively.

For spherical particles, Eq. 2 has been used to show that

$$
\begin{aligned}
F \in \mathscr{D}\left(M_{1, \alpha}\right), \alpha>n-k & \Longrightarrow F^{(n, k)} \in \mathscr{D}\left(M_{1, \alpha-(n-k)}\right), \\
F \in \mathscr{D}\left(M_{2, \alpha}\right), \alpha>0 & \Longrightarrow F^{(n, k)} \in \mathscr{D}\left(M_{2, \alpha+\frac{n-k}{2}}\right), \\
F \in \mathscr{D}\left(M_{3}\right) & \Longrightarrow F^{(n, k)} \in \mathscr{D}\left(M_{3}\right) .
\end{aligned}
$$

A proof of these results for $n-k=1$ can be found in Drees and Reiss (1992). The proof for $n-k \geq 2$ is similar.

These stability properties can be used to estimate the normalizing constants of $F^{(n, k)}$ from data in the section plane $L_{k}$ and the aim is then to get back to the normalizing constants of $F$ and predict its extremes. In a series of papers this was done in the spherical case, using a generalized gamma model for the distribution function $F$ (Takahashi and Sibuya, 1996; 1998; 2001; 2002).

A slight extension of this approach was recently obtained for spheroidal particles in $\mathbb{R}^{3}$ (Hlubinka, 2003a;b). Suppose the particles are ellipsoidal with two equal major semiaxes whose length is $X$. Denote the minor semiaxis by $W$. Then a particle is completely determined (up to position) by the bivariate variable $(X, T)$, where

$$
T=\frac{X^{2}}{W^{2}}-1
$$

is called a shape factor. Note that the spherical case is obtained for $T=0$ and deviation from spherical shape increases with $T$. Under certain isotropy and independence assumptions the observed ellipses in the section plane can be described by a size-shape variable $(Y, Z)$, where $Y$ is the length of the major semiaxis of the ellipse and $Z$ is a shape factor similarly defined as in (Eq. 16). Assuming that $(X, T)$ has a density $f(x, t)$, one can derive a relation between $f(x, t)$ and the density $g(y, z)$ of $(Y, Z)$, see Cruz-Orive (1976). Using this relationship it is possible to prove stability properties as in the spherical case assuming a fixed size or fixed shape for the spheroidal particles. A simulation study where $f(x, t)$ follows a continuous
Farlie-Gumbel-Morgenstern type of distribution can be found in Beneš et al. (2003).

Instead of considering maxima one could also be interested in minima. Stability properties for minimal radii in the spherical Wicksell corpuscle problem has been proved recently in Kötzer and Molchanov (2006).

\section{SHAPE MODELLING}

Shape modelling of planar and spatial objects with no obvious landmarks has attracted much attention in the last ten years. One approach in this direction uses the normal deformation of a sphere, which is defined via the spherical-harmonic basis on the unit sphere, see e.g., Grenander and Miller (1994); Hobolth (2003); Hobolth and Jensen (2002). This shape model can easily be generalized to higher dimensions. Thus, let $\Xi_{0} \subseteq \mathbb{R}^{n}$ be star-shaped relative to $z \in \Xi_{0}$. The boundary of $\Xi_{0}$ is determined by

$$
\left\{z+r(\omega) \omega: \omega \in S^{n-1}\right\},
$$

where $r(\omega)$ is the distance from $z$ to the boundary of $\Xi_{0}$ in direction $\omega$. We can express the radius-vector function $r(\omega)$ in terms of the spherical harmonics

$$
\left\{S_{k, m}^{(n)}(\omega): k \in \mathbb{N}_{0}, m=1, \ldots, N(n, k)\right\},
$$

which constitute an orthonormal basis on $S^{n-1}$ (Groemer, 1996; Mueller, 1966). Here $N(n, k)$ denotes the number of linearly independent spherical harmonics of degree $k$ in $n$ variables. The FourierLegendre series expansion of the radius-vector function is given by

$$
r(\omega)=\sum_{k=0}^{\infty} \sum_{m=1}^{N(n, k)} a_{k, m}^{(n)} S_{k, m}^{(n)}(\omega)
$$

where

$$
a_{k, m}^{(n)}=\int_{S^{n-1}} r(\omega) S_{k, m}^{(n)}(\omega) d \omega .
$$

For modelling shape variability, the coefficients $a_{k, m}^{(n)}$ are chosen to be random and are modelled as independent Gaussian random variables with common mean zero and variances $\lambda_{k, m}^{(n)}$,

$$
a_{k, m}^{(n)} \sim \mathbf{N}\left(0, \lambda_{k, m}^{(n)}\right), \quad k \in \mathbb{N}_{0}, m=1, \ldots, N(n, k) .
$$

Assuming moreover that

$$
\lambda_{k, m}^{(n)}=\lambda_{k}^{(n)}, \quad k \in \mathbb{N}_{0}, m=1, \ldots, N(n, k),
$$


we get, using the addition theorem for spherical harmonics,

$$
\begin{aligned}
\operatorname{Cov}( & \left.r\left(\omega_{1}\right), r\left(\omega_{2}\right)\right) \\
& =\sum_{k=0}^{\infty} \lambda_{k}^{(n)} \sum_{m=1}^{N(n, k)} S_{k, m}^{(n)}\left(\omega_{1}\right) S_{k, m}^{(n)}\left(\omega_{2}\right) \\
& =\frac{1}{\sigma_{n}} \sum_{k=0}^{\infty} \lambda_{k}^{(n)} N(n, k) P_{k}^{(n)}\left(\omega_{1} \cdot \omega_{2}\right),
\end{aligned}
$$

where $\omega_{1}, \omega_{2} \in S^{n-1}$ and $P_{k}^{(n)}(\cdot)$ denotes the Legendre polynomial of degree $k$ in $n$ variables. ¿From (Eq. 18) we see that assumption (Eq. 17) implies stationarity on the sphere, in the sense that the covariance between two points on the sphere depends only on the angle between these points. Since in (Eq. 18) $\sigma_{n}, N(n, k)$ and $P_{k}^{(n)}(\cdot)$ are known, the covariance is completely determined by the variances $\lambda_{k}^{(n)}$. Parametric models for the variances $\lambda_{k}^{(n)}$ in the planar and spatial case $(n=2,3)$ are discussed in Grenander and Miller (1994; 1998); Hobolth and Jensen (2002).

\section{OPEN PROBLEMS}

The list of geometric identities presented in this paper is not complete in the sense that it would be of great practical interest in stereological particle analysis if identities of the type (Eq. 1) could be derived for functionals $\alpha$ other than powers of volume and surface area. Such an identity is, however, only of interest in applications if it is possible to determine $\beta(X \cap T)$ from information inside the section $T$. In particular, it is important to derive identities of this type with $\alpha$ equal to squared surface area. Another class of identities still missing are those with $\alpha$ equal to intrinsic volumes in $\mathbb{R}^{n}$ or $\beta$ equal to intrinsic volumes on the sections. Some progress on the second problem has been made in Jensen and Rataj (2005) but the first problem remains open. In fact, the first problem is the one with most practical interest.

Prediction of extreme particle sizes in the case of spherical or spheroidal particles is the first step in creating a theory of "Stereology of extremes", but having applications in mind it is clearly of great importance to develop methods for particles with more complicated shape. A major problem is that only for simple particle shapes is it possible to derive relationships between the particle sizes of interest such as (Eq. 2). For example, Cruz-Orive (1976) has shown that the size distribution of ellipsoidal particles in $\mathbb{R}^{3}$ with three different semiaxes cannot be uniquely determined using information from plane sections.

\section{ACKNOWLEDGEMENTS}

This research was worked out while the author was a research fellow within the Marie Curie Training Site for Advanced Medical Imaging and Spatial Statistics (MISS), University of Aarhus, Denmark. The author would like to thank Eva B. Vedel Jensen for her great support during this fellowship. The author is also grateful to Ilya Molchanov and Adrian Baddeley for their helpful comments and suggestions.

\section{REFERENCES}

Baddeley A, Jensen EBV (2005). Stereology for Statisticians. Boca Raton: Chapman \& Hall/CRC.

Baddeley AJ (1984). Vertical sections. In: Weil W, Ambartzumian RV, eds., Stochastic geometry and stereology (Oberwolfach 1983). Berlin: Teubner.

Baddeley AJ (1985). An anisotropic sampling design. In: Nagel W, ed., Geobild '85. Jena, DDR: Wissenschaftliche Publikation, Friedrich-SchillerUniversität Jena.

Baddeley AJ (1987). Stereology and image analysis for anisotropic plane sections. In: Proceedings of the 13th International Biometric Conference, Seattle 1987. Section I-8.1.

Baddeley AJ, Gundersen HJG, Cruz-Orive LM (1986). Estimation of surface area from vertical sections. J Microsc 142:259-76.

Beneš V, Bodlák K, Hlubinka D (2003). Stereology of extremes; bivariate models and computation. Methodol Comput Appl Probab 5:289-308.

Beneš V, Rataj J (2004). Stochastic Geometry: Selected Topics. Boston: Kluwer Academic Publishers.

Blaschke W (1935a). Integralgeometrie 1. Ermittlung der Dichten für lineare Unterräume im $E_{n}$. Actualites Sci Indust 252:1-22.

Blaschke W (1935b). Integralgeometrie 2. Zu Ergebnissen von M. W. Crofton. Bull Math Soc Roumaine Sci 37:311.

Cruz-Orive LM (1976). Particle size-shape distributions: the general spheroid problem. I. Mathematical model. J Microsc 107:235-53.

Cruz-Orive LM (2005). A new stereological principle for test lines in three-dimensional space. J Microsc 219:1828.

Daley DJ, Vere-Jones D (1988). An Introduction to the Theory of Point Processes. New York: Springer Verlag.

de Haan L (1975). On regular variation and its applications to the weak convergence of sample extremes. Amsterdam: Mathematical Centre Tracts 32. 
DeHoff RT, Rhines FN (1961). Determination of number of particles per unit volume from measurements made on random plane sections: the general cylinder and the ellipsoid. Transactions AIME 221:975-82.

Drees H, Reiss RD (1992). Tail behavior in Wicksell's corpuscle problem. In: Probability Theory and Applications. Kluwer, Dordrecht.

Galambos J (1987). The Asymptotic Theory of Extreme Order Statistics (2nd edition). Florida: Krieger.

Gardner RJ (1995). Geometric tomography. New York: Cambridge University Press.

Gardner RJ, Jensen EBV, Volčič A (2003). Geometric tomography and local stereology. Adv in Appl Math 30:397-423.

Gokhale AM (1990). Unbiased estimation of curve length in 3-d using vertical slices. J Microsc 159:133-43.

Grenander U, Miller MI (1994). Representations of knowledge in complex systems (with discussion). J Roy Statist Soc Ser B 56:549-603.

Grenander U, Miller MI (1998). Computational anatomy: An emerging discipline. Quart Appl Math 5:617-94.

Groemer H (1996). Geometric applications of Fourier series and spherical harmonics. Cambridge University Press: Encyclopedia of Mathematics and its Applications, 61.

Gundersen HJG (1977). Notes on the estimation of the numerical density of arbitrary profiles: the edge effect. J Microsc 111:219-23.

Gundersen HJG (1978). Estimators of the number of objects per area unbiased by edge effects. Microscopica Acta 81:107-17.

Hanisch KH, Stoyan D (1981). Stereological estimation of the radial distribution function of centres of spheres. $\mathrm{J}$ Microsc 122:131-41.

Hlubinka D (2003a). Stereology of extremes; shape factor of spheroids. Extremes 6:5-24.

Hlubinka D (2003b). Stereology of extremes; size of spheroids. Mathematica Bohemica 128:419-38.

Hobolth A (2003). The spherical deformation model. Biostatistics 4:583-95.

Hobolth A, Jensen EBV (2002). Stereological analysis of shape. Image Anal Stereol 21:23-9.

Jensen EBV (1998). Local Stereology. Singapore. World Scientific Publishing.

Jensen EBV, Kiêu K (1992). A new integral geometric formula of the Blaschke-Petkantschin type. Math Nachr 156:57-74.

Jensen EBV, Kiêu K (1994). Unbiased stereological estimation of $d$-dimensional volume in $\mathbb{R}^{n}$ from an isotropic random slice through a fixed point. Adv Appl Prob 26:1-12.
Jensen EBV, Pawlas Z (2005). Stereological estimation of the particle volume distribution without shape assumptions. In preparation .

Jensen EBV, Rataj J (2005). A rotational integral formula for intrinsic volumes. In preparation .

Kötzer S, Molchanov I (2006). On the domain of attraction for the lower tail in Wicksell's corpuscle problem. In: Proceedings of the 6th International Conference on Stereology, Spatial Statistics and Stochastic Geometry $S^{4} G$, Prague 2006.

Matheron G (1975). Random sets and integral geometry. New York: John Wiley and Sons.

Mecke J, Stoyan D (1980). Stereological problems for spherical particles. Math Nachr 96:311-7.

Miles RE (1974). On the elimination of edge effects in planar sampling. In: Stochastic Geometry: a tribute to the memory of Rollo Davidson. New York: Wiley.

Miles RE (1979). Some new integral geometric formulae, with stochastic applications. J Appl Prob 16:592-606.

Møller J (1987). A simple derivation of a formula of blaschke and petkantschin. Research Report Department of Theoretical Statistics Institute of Mathematics University of Aarhus 138.

Mueller C (1966). Spherical harmonics. Berlin: Springer.

Nielsen J (1999). The distribution of volume reductions induced by isotropic random projections. Adv Appl Prob 31:985-94.

Petkantschin B (1936). Integralgeometrie 6. Zusammenhänge zwischen den Dichten der linearen Unterräume im $n$-dimensionalen Raum. Abh Math Sem Univ Hamburg 11:249-310.

Schneider R, Weil W (2000). Stochastische Geometrie. Stuttgart: Teubner.

Stoyan D (1979). Proofs of some fundamental formulae of stereology for non-Poisson grain models. Math Operationsforsch Statist Ser Optim 10:573-81.

Stoyan D (1982). Stereological formulae for size distributions via marked point processes. Probab Math Statist 2:161-6.

Stoyan D (1990). Stereology and stochastic geometry. International Statistical Review 58:227-42.

Stoyan D (1996). Statistical analysis of random particles. Z Angew Math Mech 76:315-8.

Stoyan D, Kendall WS, Mecke J (1987). Stochastic Geometry and its Applications. Chichester: Wiley.

Stoyan D, Mecke J (1983). Stochastische Geometrie: eine Einführung. Berlin: Akademie-Verlag.

Takahashi R, Sibuya M (1996). The maximum size of the planar sections of random spheres and its application to metallurgy. Ann Inst Statist Math 48:127-44. 
Takahashi R, Sibuya M (1998). Prediction of the maximum size in Wicksell's corpuscle problem. Ann Inst Statist Math 50:361-77.

Takahashi R, Sibuya M (2001). Prediction of the maximum size in Wicksell's corpuscle problem, II. Ann Inst Statist Math 53:647-60.

Takahashi R, Sibuya M (2002). Metal fatigue, Wicksell transform and extreme values. Appl Stoch Models Bus Ind 18:301-12.

Weibel ER (1979). Stereological Methods, 1. Practical Methods for Biological Morphometry. London:
Academic Press.

Weibel ER (1980). Stereological Methods, 2. Theoretical Foundations. London: Academic Press.

Weil W (1983). Stereology: a survey for geometers. In: Gruber P, Wills J, eds., Convexity and its Applications. Basel, Boston, Stuttgart: Birkhäuser, 360-412.

Wicksell SD (1925). The corpuscle problem. A mathematical study of a biometric problem. Biometrika 17:84-99.

Zähle M (1990). A kinematic formula and moment measures of random sets. Math Nachr 149:325-40. 